Research Paper

\title{
Association of sirtuins with clinicopathological parameters and overall survival in gastric cancer
}

\author{
Xiaobing Shen ${ }^{1,}{ }^{,}$, Pengfei Li ${ }^{1,}{ }^{*}$, Yuchao Xu ${ }^{1}$, Xiaowei Chen ${ }^{1}$, Haixiang Sun ${ }^{1}$, Ying Zhao ${ }^{1}$, \\ Mengqi Liu ${ }^{1}$ and Wenwen Zhang ${ }^{1}$ \\ ${ }^{1}$ Key Laboratory of Environmental Medicine Engineering, Ministry of Education, School of Public Health, Southeast University, \\ Nanjing, 210009, China \\ *These authors contributed equally to this work and should be considered as co-first authors
}

Correspondence to: Xiaobing Shen, email: xbshen_seu@163.com

Keywords: sirtuins, overall survival, kaplan-meier plotter, gastric cancer

Received: November 22, $2016 \quad$ Accepted: August 23, $2017 \quad$ Published: September 08, 2017

Copyright: Shen et al. This is an open-access article distributed under the terms of the Creative Commons Attribution License 3.0 (CC BY 3.0), which permits unrestricted use, distribution, and reproduction in any medium, provided the original author and source are credited.

\section{ABSTRACT}

To evaluate the associations of sirtuins (SIRT1-7) with clinicopathological parameters in gastric cancer, sirtuins expression profile in NCBI GEO datasets, GSE62254 and GSE15459, was integrated and analyzed. The results suggested that SIRT4, SIRT6, and SIRT7 were associated with Lauren classification and SIRT3-5 were associated with pStage in gastric cancer. Then an online database derived from 1,065 gastric cancer cases, Kaplan-Meier plotter, was used to explore the associations of the mRNA levels of sirtuins with overall survival in gastric cancer. Survival curves generated from Kaplan-Meier plotter suggested that high expression of SIRT1 mRNA was favorable for overall survival in gastric cancer (SIRT1: HR = 0.64, 95\% CI = 0.54-0.76, $P=2.2 E-07$ ), high expressions of SIRT2-4 and SIRT6-7 were poor for overall survival (SIRT2: HR = 2.31, 95\% CI = 1.87-2.87, $P=3.6 E-15$; SIRT3: HR = $1.99,95 \%$ CI $=1.62-2.45, P=2.6 E-11 ;$ SIRT4: HR $=1.41,95 \%$ CI $=1.19-1.68, P=$ 6.6E-05; SIRT6: HR = 2.02, 95\% CI = 1.66-2.47, $P=1.7 \mathrm{E}-12$; SIRT7: HR = 1.96, 95\% $C I=1.63-2.35, P=2.7 E-13)$, whereas no significant association existed between SIRT5 mRNA expression and overall survival. Further analyses stratified by gender, stages, Lauren classification, differentiation, treatment, and HER2 status were also performed. In summary, high SIRT1 mRNA level was associated with better overall survival, SIRT2-4 and 6-7 were associated with poor overall survival, whereas SIRT5 did not show significant association with overall survival in gastric cancer.

\section{INTRODUCTION}

Gastric cancer remains the major cause of cancerrelated death with a bad prognosis [1-3]. Despite recent efforts in multimodal treatment approaches, approximately half of patients diagnosed with advanced gastric cancer still die from recurrent disease after surgical resection or distant metastasis [4, 5]. Now it has been recognized that multiple genetic and epigenetic alterations or abnormality occur in the development of GC [6]. Thus, the identification on the mechanism of initiation, progression, as well as investigation of differential diagnostic prognostic marker and potential drug target, is still needed and will help to provide better prognosis and individualized treatments.

The sirtuins are a family of proteins homologous to yeast silent information regulator 2 (Sir2) and widely expressed in normal tissues in mammary animals. Up to now, seven members have been identified in human (SIRT1-7) and possess nicotinamide adenine dinucleotide (NAD+)-dependent lysine deacetylase (SIRT1, SIRT2, SIRT3, SIRT5, SIRT6, and SIRT7) and mono-ribosyltransferase (SIRT4 and SIRT6) activities [7-14]. Recently, SIRT5 was shown to be a NAD+-dependent protein lysine demalonylase and desuccinylase [15]. The sirtuins play essential roles in 
cellular physiology including cell metabolism, cell cycle, cell division, and transcriptional regulation and are also involved in the pathogenesis of series of diseases such as metabolic diseases [16], neurodegenerative diseases [17], cardiovascular diseases [18], and aging [19]. Some previous studies have been performed to explore the clinical value and mechanism of sirtuins in gastric cancer. However, the results are inconsistent and not all the seven sirtuins are investigated and compared.

Kaplan-Meier plotter (KM plotter), an online tool, can be utilized to analyze correlations of individual genes with prognosis of patients. This database was initially established using data from a group of 1,809 breast cancer patients $[20,21]$. Later, this database also included gene expression data of a total of 1,065 gastric cancer patients derived from NCBI GEO datasets GSE14210, GSE15459, GSE22377, GSE29272, GSE51105, and GSE62254. In this study, we used datasets GSE15459 and GSE62254 to explore the associations of sirtuins with clinicopathological parameters and used KM plotter database to determine the prognostic role of individual sirtuins in human gastric cancer.

\section{RESULTS}

\section{Correlations of sirtuins with clinicopathological parameters in GC}

The associations of sirtuins with the clinicopathological parameters of the patients with GC were firstly explored. We downloaded the datasets, GSE62254 and GSE15459, from NCBI GEO database. Both datasets were consisted of relative large cohort of GC patients and have also been included in the KM plotter database. In GSE62254, SIRT1 and SIRT2 exerted no associations with the clinical features, SIRT3 was associated with T stage, SIRT4 was associated with pStage, SIRT5 was associated with T stage, SIRT6 was associated with age, M stage, and pStage, and SIRT7 was associated with Lauren classification, N stage, and pStage (Table 1). In GSE15459, significant associations were found between Lauren classification and SIRT7, pStage and SIRT2, and pStage and SIRT3 (Table 2). In addition, GSE62254 and GSE15459 were also integrated to investigate the correlations of sirtuins with clinicopathological parameters in GC. The results suggested SIRT4, SIRT6, and SIRT7 were associated with Lauren classification, and SIRT3-5 were associated with pStage (Table 3). Meanwhile, the KM plotter database also included gene expression profile of normal/adjacent tissue from 57 cases. Compare with the normal/adjacent tissues, SIRT2-6 expression in GC cancer tissues was lower while SIRT1 and SIRT7 had no difference (Supplementary Table 1). However, the controls were rare and not each separate dataset was consisted of controls, and the results should further verified.

\section{Correlations of sirtuins with $\mathrm{OS}$ in GC in overall}

In overall, $876 \mathrm{GC}$ cases in $\mathrm{KM}$ plotter database were available to investigate the correlations of SIRT1-4 and SIRT6-7 with OS while 631 cases were available for SIRT1. Auto select best cutoff value was used to split the patients in survival analyses. Survival curves suggested that high expression of SIRT1 mRNA was favorable for OS (SIRT1: $\mathrm{HR}=0.64,95 \% \mathrm{CI}=0.54-0.76, P=2.2 \mathrm{E}-$ 07), high expressions of SIRT2-4 and SIRT6-7 were poor for OS (SIRT2: $\mathrm{HR}=2.31,95 \% \mathrm{CI}=1.87-2.87, P=3.6 \mathrm{E}-$ 15; SIRT3: $\mathrm{HR}=1.99,95 \% \mathrm{CI}=1.62-2.45, P=2.6 \mathrm{E}-11$; SIRT4: $\mathrm{HR}=1.41,95 \% \mathrm{CI}=1.19-1.68, P=6.6 \mathrm{E}-05$; SIRT6: $\mathrm{HR}=2.02,95 \% \mathrm{CI}=1.66-2.47, P=1.7 \mathrm{E}-12$; SIRT7: $\mathrm{HR}=1.96,95 \% \mathrm{CI}=1.63-2.35, P=2.7 \mathrm{E}-13)$, and no significant association existed between SIRT5 and OS in GC (Figure 1 and Table 4).

Then we performed subgroup analyses to explore the effects of clinicopathological features, such as gender, stage, Lauren classification, differentiation, treatment, and HER2 status, on the associations between sirtuins mRNA expression and OS in GC. The effects of gender were firstly investigated. There were 545 male GC patients available for analyzing the correlations between SIRT1-4 and 6-7 and OS while there were 349 male GC patients available for SIRT5. And 236 female patients were available for SIRT1-4 and 6-7 and 187 female patients for SIRT5. Either in male patients or female patients, SIRT14 and SIRT6-7 were associated with OS while SIRT5 was not significantly associated with OS (Table 5).

Secondly, subgroup analyses according to stage were performed. In patients with stage 1 cancer, significant association was only found between SIRT2 and OS ( $n$ $=67$, Table 6). In stage 2, SIRT2, 3, 4, 6, and 7 were correlated with OS $(n=140)$. In stage 3 , all sirtuins were correlated with OS ( $n=305$ for SIRT1-4 and 6-7, $n=$ 197 for SIRT5). And in stage 4, significant association was identified between SIRT2, 5, 6, and 7 and OS $(n=$ 140). Notably, unlike the results in overall, SIRT5 was significantly associated with OS in stage 3 and stage 4, however, exerting opposite effects on OS (stage 3, $\mathrm{n}=197$, $\mathrm{HR}=1.70,95 \% \mathrm{CI}=1.16-2.49, P=6.3 \mathrm{E}-03$; stage $4, n=$ $140, \mathrm{HR}=0.63,95 \% \mathrm{CI}=0.42-0.94, P=2.2 \mathrm{E}-02)$.

Thirdly, Lauren classification was used to stratify the patients. SIRT1, 2, 3, 6, and 7 were found to be associated with OS in intestinal GC $(n=320$, Table 7$)$. SIRT2, 3 , and 4 were associated with OS in diffuse GC $(n=141)$. And, only SIRT3 was associated with OS in mixed GC $(n=32)$.

Furthermore, subgroup analyses according to differentiation or treatment were also performed, respectively. In $\mathrm{GC}$ with poor differentiation, significant associations were identified between SIRT4-5 and OS ( $n=165$ for SIRT4; $n=121$ for SIRT5, Table 8). And significant association was only found between SIRT3 and $\mathrm{OS}$ in GC with moderated differentiation $(n=67)$. In GC with well differentiation, SIRT3 and 7 was associated 
with OS $(n=32)$. For association between SIRT5 and OS in GC with well differentiation, analysis could not be conducted due to the case number was very small ( $n$ $=5$ ). After grouping by treatment (surgery alone, 5-FU adjuvant therapy, or other adjuvant therapy), significant associations were found between SIRT2-4 and OS in GC patients underwent surgery alone ( $n=380$, Table 9). In patients underwent 5-FU adjuvant therapy, significant associations were found between SIRT1, 2, 5, and 7 and OS $(n=153$, Table 9). And significant associations were found between SIRT6 and OS in patients underwent other adjuvant therapy ( $n=76$, Table 9$)$.

Finally, effects of HER2 status on the associations between sirtuins and $\mathrm{OS}$ in $\mathrm{GC}$ were evaluated. In GC with negative HER2 status, all sirtuins except SIRT5 and SIRT7 were associated with OS $(n=532$, Table 10$)$ and in
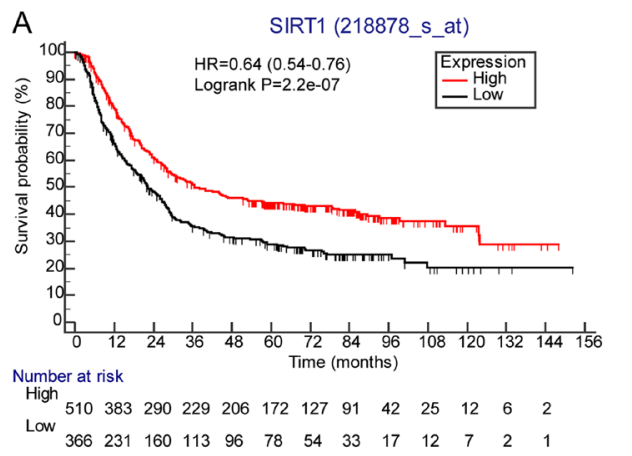

C

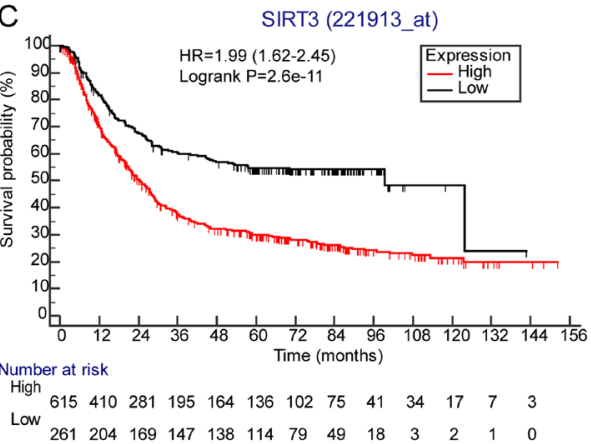

E

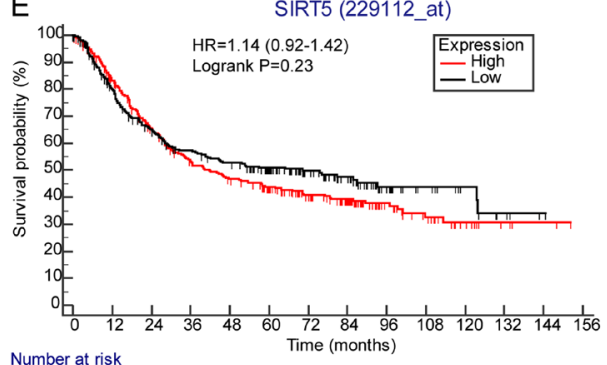
Number at risk

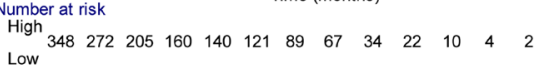

Low $\begin{array}{llllllllllllll}283 & 210 & 166 & 140 & 127 & 101 & 77 & 51 & 25 & 15 & 9 & 4 & 1\end{array}$

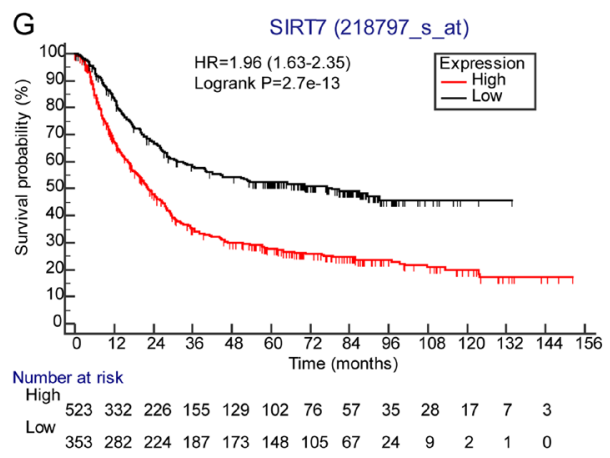

B SIRT2 (220605_s_at)

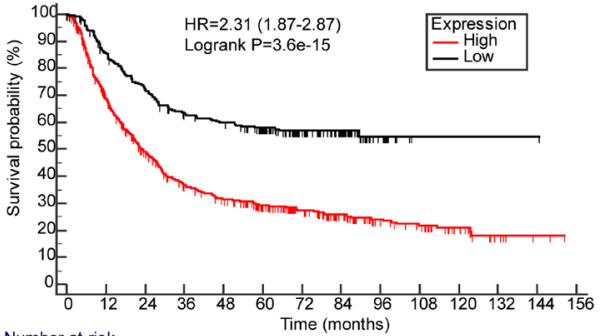

Number at risk

High $623 \quad 407 \quad 276 \begin{array}{llllllllll}195 & 164 & 133 & 103 & 75 & 44 & 36 & 18 & 7 & 2\end{array}$

Low $25 \begin{array}{lllllllllllll}253 & 207 & 174 & 147 & 138 & 117 & 78 & 49 & 15 & 1 & 1 & 1 & 1\end{array}$

D

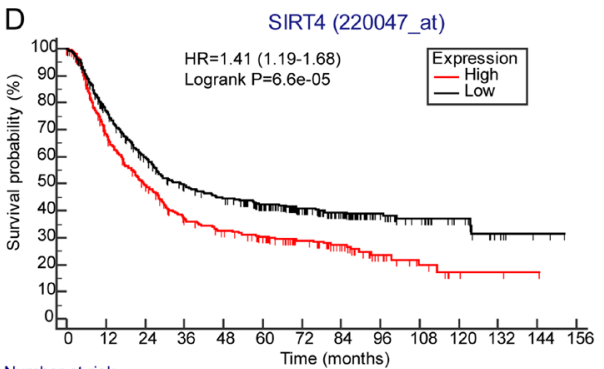

Number at risk

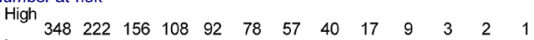

Low $\begin{array}{lllllllllllll}528 & 392 & 294 & 234 & 210 & 172 & 124 & 84 & 42 & 28 & 16 & 6 & 2\end{array}$

F

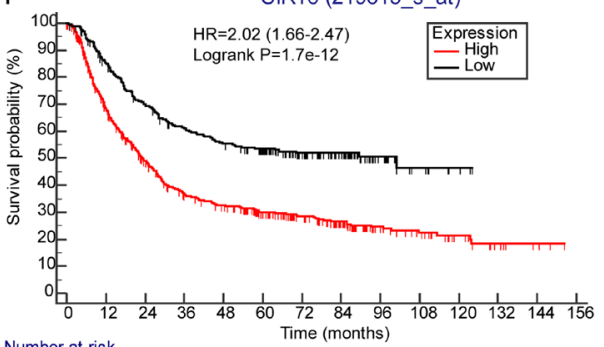

Number at risk

High $\begin{array}{rllllllllllll}597 & 387 & 265 & 185 & 159 & 126 & 95 & 67 & 38 & 29 & 16 & 8 & 3\end{array}$

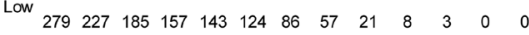

Figure 1: Correlations of sirtuins mRNA expressions with OS in all GC patients. (A) SIRT1 $(n=876)$. (B) SIRT2 $(n=876)$. (C) SIRT3 $(n=876)$. (D) SIRT4 $(n=876)$. (E) SIRT5 $(n=631)$. (F) SIRT6 $(n=836)$. (G) SIRT7 $(n=836)$. 
Table 1: Sirtuins and clinicopathological parameters in patients with GC (GSE62254)

\begin{tabular}{|c|c|c|c|c|c|c|c|c|}
\hline Parameters & Cases & SIRT1+ & SIRT2+ & SIRT3+ & SIRT4+ & SIRT5+ & SIRT6+ & SIRT7+ \\
\hline \multicolumn{9}{|l|}{ Gender } \\
\hline Male & 199 & 98 & 96 & 102 & 93 & 98 & 93 & 101 \\
\hline Female & 101 & 52 & 54 & 48 & 57 & 52 & 57 & 49 \\
\hline \multicolumn{9}{|l|}{ Age } \\
\hline$<65$ & 161 & 78 & 82 & 78 & 87 & 79 & $70^{*}$ & 81 \\
\hline$\geq 65$ & 139 & 72 & 68 & 72 & 63 & 71 & 80 & 61 \\
\hline \multicolumn{9}{|c|}{ Lauren classification } \\
\hline Intestinal & 150 & 75 & 75 & 85 & 69 & 82 & 83 & $85^{*}$ \\
\hline Diffuse & 142 & 71 & 72 & 62 & 78 & 64 & 62 & 64 \\
\hline Mixed & 8 & 4 & 3 & 3 & 3 & 4 & 5 & 1 \\
\hline \multicolumn{9}{|l|}{ T stage } \\
\hline 2 & 188 & 88 & 93 & $105^{*}$ & 93 & $104 *$ & 100 & 102 \\
\hline 3 & 91 & 49 & 47 & 34 & 50 & 36 & 38 & 37 \\
\hline 4 & 21 & 13 & 10 & 11 & 7 & 10 & 12 & 11 \\
\hline \multicolumn{9}{|l|}{ N stage } \\
\hline 0 & 38 & 20 & 19 & 18 & 16 & 19 & 24 & $10^{*}$ \\
\hline 1 & 131 & 58 & 60 & 63 & 72 & 71 & 65 & 75 \\
\hline 2 & 80 & 46 & 43 & 44 & 42 & 42 & 33 & 42 \\
\hline 3 & 51 & 26 & 28 & 25 & 20 & 18 & 28 & 23 \\
\hline \multicolumn{9}{|l|}{ M stage } \\
\hline 0 & 273 & 139 & 133 & 138 & 139 & 137 & $143^{*}$ & 133 \\
\hline 1 & 27 & 11 & 17 & 12 & 11 & 13 & 7 & 17 \\
\hline \multicolumn{9}{|l|}{ pStage } \\
\hline 1 & 30 & 14 & 14 & 17 & $11^{*}$ & 15 & $22 *$ & $8^{*}$ \\
\hline 2 & 97 & 42 & 46 & 50 & 56 & 55 & 49 & 57 \\
\hline 3 & 96 & 53 & 49 & 47 & 52 & 49 & 42 & 47 \\
\hline 4 & 77 & 41 & 41 & 36 & 77 & 31 & 37 & 38 \\
\hline
\end{tabular}

$* P<0.05$ demonstrated by Chi-square test.

GC with positive HER2 status, all sirtuins except SIRT4 were associated with OS ( $n=344$ for SIRT1-4 and 6-7; $n=202$ for SIRT5).

In addition, the online database also included the first progression (FP) information of the GC patients, and the analyses results suggested that high expression of SIRT1 was associated with long FP time while high expression of SIRT2-4 and SIRT6-7 was associated with short FP time and no significant association was found between SIRT5 and FP in GC in overall (Supplementary Figure 1 and Supplementary Table 2).

\section{DISCUSSION}

Gastric cancer (GC) is main cause of cancer related death and presents high mortality rate among all digestive tract malignancies due to chemoradiotherapy resistance and distant metastasis. Thus it is crucial to reveal pathogenesis of GC and find novel prognostic strategies, early diagnostic tools, and effective therapeutic approaches. Here, we used an online database to explore the clinical value of sirtuins in predicting overall survival (OS) in GC.

Of all the seven sirtuins, SIRT1 was the most studied one in GC. A series of studies have investigated the associations of SIRT1 with OS in GC. Of which, four studies reported that high SIRT1 expression was associated with poor OS [22-25] while one study reported that there was a trend of association between SIRT1 with good OS [26]. A meta-analysis combing the previous studies suggested that high SIRT1 expression was closely linked with the 3 -year OS $(\mathrm{OR}=0.25,95 \%$ 
Table 2: Sirtuins and clinicopathological parameters in patients with GC (GSE15459)

\begin{tabular}{|c|c|c|c|c|c|c|c|c|}
\hline Parameter & Cases & SIRT1+ & SIRT2+ & SIRT3+ & SIRT4+ & SIRT5+ & SIRT6+ & SIRT7+ \\
\hline \multicolumn{9}{|l|}{ Gender } \\
\hline Male & 125 & 63 & 67 & 65 & 66 & 59 & 66 & 63 \\
\hline Female & 67 & 33 & 29 & 31 & 30 & 37 & 30 & 33 \\
\hline \multicolumn{9}{|l|}{ Age } \\
\hline$<65$ & 84 & 42 & 38 & 47 & 40 & 41 & 45 & 42 \\
\hline$\geq 65$ & 108 & 54 & 58 & 49 & 56 & 55 & 51 & 54 \\
\hline \multicolumn{9}{|c|}{ Lauren classification } \\
\hline Intestinal & 99 & 43 & 49 & 43 & 50 & 49 & 54 & $60 *$ \\
\hline Diffuse & 75 & 42 & 39 & 45 & 41 & 38 & 33 & 28 \\
\hline Mixed & 18 & 11 & 8 & 8 & 5 & 9 & 9 & 8 \\
\hline \multicolumn{9}{|l|}{ pStage } \\
\hline 1 & 31 & 17 & $24 *$ & $22 *$ & 17 & 19 & 15 & 17 \\
\hline 2 & 29 & 17 & 15 & 15 & 16 & 19 & 15 & 13 \\
\hline 3 & 72 & 32 & 31 & 39 & 30 & 35 & 39 & 38 \\
\hline 4 & 60 & 30 & 26 & 20 & 33 & 23 & 27 & 28 \\
\hline
\end{tabular}

$* P<0.05$ demonstrated by Chi-square test.

Table 3: Sirtuins and clinicopathological parameters in patients with GC (integrated analysis of GSE62254 and GSE15459)

\begin{tabular}{|c|c|c|c|c|c|c|c|c|}
\hline Parameters & Cases & SIRT1+ & SIRT2+ & SIRT3+ & SIRT4+ & SIRT5+ & SIRT6+ & SIRT7+ \\
\hline \multicolumn{9}{|l|}{ Gender } \\
\hline Male & 324 & 161 & 163 & 167 & 159 & 157 & 159 & 164 \\
\hline Female & 168 & 85 & 83 & 79 & 87 & 89 & 87 & 82 \\
\hline \multicolumn{9}{|l|}{ Age } \\
\hline$<65$ & 245 & 120 & 120 & 125 & 127 & 120 & 115 & 123 \\
\hline$\geq 65$ & 247 & 126 & 126 & 121 & 119 & 126 & 131 & 115 \\
\hline \multicolumn{9}{|c|}{ Lauren classification } \\
\hline Intestinal & 249 & 118 & 124 & 128 & $119^{*}$ & 131 & $137 *$ & $145^{*}$ \\
\hline Diffuse & 217 & 113 & 111 & 107 & 119 & 102 & 95 & 92 \\
\hline Mixed & 26 & 15 & 11 & 11 & 8 & 13 & 14 & 9 \\
\hline \multicolumn{9}{|l|}{ pStage } \\
\hline 1 & 61 & 31 & 38 & $39 *$ & $28 *$ & $34 *$ & 37 & 25 \\
\hline 2 & 126 & 59 & 61 & 65 & 72 & 74 & 64 & 70 \\
\hline 3 & 168 & 85 & 80 & 86 & 82 & 84 & 81 & 85 \\
\hline 4 & 137 & 71 & 67 & 56 & 110 & 54 & 64 & 66 \\
\hline
\end{tabular}

$* P<0.05$ demonstrated by Chi-square test.

Table 4: Correlations of sirtuins with OS in GC in overall

\begin{tabular}{lcccc}
\hline Sirtuins & Cases & HR & $\mathbf{9 5 \%}$ CI & $\boldsymbol{P}$-value \\
\hline SIRT1 & 876 & $\mathbf{0 . 6 4}$ & $\mathbf{0 . 5 4 - 0 . 7 6}$ & $\mathbf{2 . 2 E - 0 7}$ \\
SIRT2 & 876 & $\mathbf{2 . 3 1}$ & $\mathbf{1 . 8 7 - 2 . 8 7}$ & $\mathbf{3 . 6 E - 1 5}$ \\
SIRT3 & 876 & $\mathbf{1 . 9 9}$ & $\mathbf{1 . 6 2 - 2 . 4 5}$ & $\mathbf{2 . 6 E - 1 1}$ \\
SIRT4 & 876 & $\mathbf{1 . 4 1}$ & $\mathbf{1 . 1 9 - 1 . 6 8}$ & $\mathbf{6 . 6 E - 0 5}$ \\
SIRT5 & 631 & 1.14 & $0.92-1.42$ & $2.3 \mathrm{E}-01$ \\
SIRT6 & 876 & $\mathbf{2 . 0 2}$ & $\mathbf{1 . 6 6}-\mathbf{2 . 4 7}$ & $\mathbf{1 . 7 E - 1 2}$ \\
SIRT7 & 876 & $\mathbf{1 . 9 6}$ & $\mathbf{1 . 6 3 - 2 . 3 5}$ & $\mathbf{2 . 7 E - 1 3}$ \\
\hline
\end{tabular}


Table 5: Correlations of sirtuins with OS in GC stratified by gender

\begin{tabular}{|c|c|c|c|c|c|}
\hline Sirtuins & Gender & Cases & HR & $95 \% \mathrm{CI}$ & $P$-value \\
\hline \multirow[t]{2}{*}{ SIRT1 } & Male & 545 & 0.45 & $0.31-0.66$ & $1.7 \mathrm{E}-05$ \\
\hline & Female & 236 & 0.63 & $0.51-0.77$ & $1.3 \mathrm{E}-05$ \\
\hline \multirow[t]{2}{*}{ SIRT2 } & Male & 545 & 2.08 & $1.38-3.14$ & $3.5 \mathrm{E}-04$ \\
\hline & Female & 236 & 2.62 & $2.02-3.40$ & $6.2 \mathrm{E}-14$ \\
\hline \multirow[t]{2}{*}{ SIRT3 } & Male & 545 & 2.12 & $1.41-3.18$ & 2.1E-04 \\
\hline & Female & 236 & 2.07 & $1.60-2.67$ & $1.8 \mathrm{E}-08$ \\
\hline \multirow[t]{2}{*}{ SIRT4 } & Male & 545 & 1.88 & $1.33-2.68$ & 3.3E-04 \\
\hline & Female & 236 & 1.46 & $1.16-1.84$ & $1.4 \mathrm{E}-03$ \\
\hline \multirow[t]{2}{*}{ SIRT5 } & Male & 349 & 0.76 & $0.48-1.20$ & 2.3E-01 \\
\hline & Female & 187 & 0.80 & $0.57-1.12$ & $2.0 \mathrm{E}-01$ \\
\hline \multirow[t]{2}{*}{ SIRT6 } & Male & 545 & 2.24 & $1.41-3.55$ & 4.4E-04 \\
\hline & Female & 236 & 2.26 & $1.75-2.92$ & $1.6 \mathrm{E}-10$ \\
\hline \multirow[t]{2}{*}{ SIRT7 } & Male & 545 & 1.96 & $1.38-2.79$ & $1.2 \mathrm{E}-04$ \\
\hline & Female & 236 & 2.12 & $1.68-2.67$ & $6.6 \mathrm{E}-11$ \\
\hline
\end{tabular}

$\mathrm{CI}=0.16-0.39, P<0.00001$, fixed model $)$ while it was not associated with 5 -year $\mathrm{OS}(\mathrm{OR}=0.44,95 \% \mathrm{CI}=$ $0.15-1.28, P=0.13$, random model) [27]. In our study, we found that high expression of SIRT1 mRNA was favorable for OS (SIRT1: $\mathrm{HR}=0.64,95 \% \mathrm{CI}=0.54-0.76, P=2.2 \mathrm{E}-$ 07). The inconsistency of these results might be due to the heterogeneity of these studies such as sample size, cancer site, and cutoff value. Several meta-analyses have also been performed to evaluate the associations of SIRT1 with OS in other solid carcinomas. All the meta-analyses studies revealed a significant association between high SIRT1 with poor OS in these solid carcinomas including breast cancer [28], hepatocellular carcinoma [29], colorectal cancer [30], lung cancer [31, 32], liver cancer [32]. Wang et al. also have pooled all the eligible data of solid malignancies and identified the same conclusion [32]. Mechanism researches suggested that SIRT1 can counteract the activation of STAT3 and NF- $\mathrm{KB}$ to repress cell growth [33] and lead to G1-phase arrest via NF- $\mathrm{kB} /$ Cyclin D1 signaling in gastric cancer[34]. The previous studies suggested that SIRT1 was also the target of certain microRNAs such as miR-543 and miR-204. miR-543 can promote gastric cancer cell proliferation by targeting SIRT1[35] and miR-204 can down-regulate SIRT1 and revert SIRT1-induced epithelial-mesenchymal transition, anoikis resistance, and invasion in gastric cancer[36].

For SIRT2, a previous study indicated that combination of SIRT2 with other three genes could be used to predict OS in GC[37]. Here, we identified that high expression of SIRT2 expression was a biomarker of worse $\mathrm{OS}$ in $\mathrm{GC}$ patients $(\mathrm{HR}=2.31,95 \% \mathrm{CI}=1.87-2.87$, $P=3.6 \mathrm{E}-15)$.

Up to now, there were three studies reporting the association of SIRT3 with OS in GC. The association was significant in two studies $[38,39]$ and insignificant in one study [40]. A meta-analysis has pooled the previous two studies and found increased SIRT3 expression was associated with better OS in GC, however, the total included cases were only 286 [41]. Our results suggested that high SIRT3 mRNA expression was associated with poor prognosis in $\mathrm{GC}(\mathrm{HR}=1.99,95 \% \mathrm{CI}=1.62-2.45$, $P=2.6 \mathrm{E}-11)$. The inconsistency needed to be verified in further studies with large scale of patients. Function studies revealed that SIRT3 plays dual role in GC development. Wang et al. reported that SIRT3 can inhibit cell proliferation in human gastric cancer through downregulation of Notch-1 [42] whereas Cui et al. reported that SIRT3 can enhance glycolysis and proliferation in SIRT3expressing GC Cells [43].

The study performed by Huang et al. suggested SIRT4 is associated with some clinicopathological features in GC but did not reported the association of SIRT4 with prognosis [44]. In the present study, we found that high SIRT4 mRNA expression exhibited a significant associations with Lauren classification, pStage, and OS in GC (OS: $\mathrm{HR}=1.41,95 \% \mathrm{CI}=1.19-1.68, P=6.6 \mathrm{E}-05)$.

Currently, SIRT5 and 6 have not been investigated in gastric cancer, but have been shown to be involved in other cancers, such as breast cancer, lung cancer, hepatocellular carcinoma, head and neck squamous cell carcinoma, and ovarian cancer [45-50]. Our study revealed that high SIRT6 expression was associated with poor OS in GC (HR $=2.02,95 \% \mathrm{CI}=1.66-2.47, P=1.7 \mathrm{E}-12)$ while SIRT5 was not correlated with OS $(\mathrm{HR}=1.14,95 \% \mathrm{CI}=0.92-$ $1.42, P=0.23$ ). Interestingly, after stratification by stage or differentiation, high SIRT5 mRNA expression was associated with $\mathrm{OS}$ in stage $3(\mathrm{HR}=1.70,95 \% \mathrm{CI}=1.16$ $2.49, P=6.3 \mathrm{E}-03)$, stage $4(\mathrm{HR}=0.63,95 \% \mathrm{CI}=0.42$ 
Table 6: Correlations of sirtuins with OS in GC stratified by stages

\begin{tabular}{|c|c|c|c|c|c|}
\hline Sirtuins & Stage & Cases & HR & $95 \% \mathrm{CI}$ & $P$-value \\
\hline \multirow[t]{4}{*}{ SIRT1 } & 1 & 67 & 0.38 & $0.14-1.06$ & $5.6 \mathrm{E}-02$ \\
\hline & 2 & 140 & 1.55 & $0.78-3.06$ & $2.1 \mathrm{E}-01$ \\
\hline & 3 & 305 & 0.59 & $0.43-0.81$ & 9.4E-04 \\
\hline & 4 & 148 & 0.81 & $0.55-1.19$ & $2.8 \mathrm{E}-01$ \\
\hline \multirow[t]{4}{*}{ SIRT2 } & 1 & 67 & 4.71 & $1.33-16.74$ & $8.5 E-03$ \\
\hline & 2 & 140 & 2.55 & $1.37-4.76$ & 2.3E-03 \\
\hline & 3 & 305 & 2.60 & $1.79-3.77$ & 2.1E-07 \\
\hline & 4 & 148 & 1.65 & $1.11-2.46$ & 1.3E-02 \\
\hline \multirow[t]{4}{*}{ SIRT3 } & 1 & 67 & 11.02 & $1.44-84.40$ & 3.9E-03 \\
\hline & 2 & 140 & 3.36 & $1.69-6.70$ & 2.5E-04 \\
\hline & 3 & 305 & 2.21 & $1.52-3.21$ & $1.8 \mathrm{E}-05$ \\
\hline & 4 & 148 & 0.76 & $0.50-1.14$ & $1.8 \mathrm{E}-01$ \\
\hline \multirow[t]{4}{*}{ SIRT4 } & 1 & 67 & 1.88 & $0.70-5.08$ & $2.1 \mathrm{E}-01$ \\
\hline & 2 & 140 & 2.25 & $1.22-4.18$ & 8.1E-03 \\
\hline & 3 & 305 & 1.43 & $1.08-1.90$ & $1.3 \mathrm{E}-02$ \\
\hline & 4 & 148 & 1.32 & $0.89-1.97$ & $1.6 \mathrm{E}-01$ \\
\hline \multirow[t]{4}{*}{ SIRT5 } & 1 & 62 & 3.00 & $0.66-13.58$ & $1.3 \mathrm{E}-01$ \\
\hline & 2 & 135 & 1.91 & $0.91-4.03$ & $8.1 \mathrm{E}-02$ \\
\hline & 3 & 197 & 1.70 & $1.16-2.49$ & $6.3 \mathrm{E}-03$ \\
\hline & 4 & 140 & 0.63 & $0.42-0.94$ & 2.1E-02 \\
\hline \multirow[t]{4}{*}{ SIRT6 } & 1 & 67 & 2.63 & $0.083-8.29$ & 8.7E-02 \\
\hline & 2 & 140 & 2.69 & $1.42-5.12$ & 1.7E-03 \\
\hline & 3 & 305 & 1.90 & $1.42-2.54$ & 1.0E-05 \\
\hline & 4 & 148 & 1.49 & $1.02-2.2$ & $3.9 \mathrm{E}-02$ \\
\hline \multirow[t]{4}{*}{ SIRT7 } & 1 & 67 & 2.76 & $0.94-8.08$ & $5.4 \mathrm{E}-02$ \\
\hline & 2 & 140 & 2.03 & $1.11-3.72$ & $1.9 \mathrm{E}-02$ \\
\hline & 3 & 305 & 1.81 & $1.33-2.46$ & 1.3E-04 \\
\hline & 4 & 148 & 1.60 & $1.05-2.45$ & $2.8 \mathrm{E}-02$ \\
\hline
\end{tabular}

$0.94)$, and poor differentiated $\mathrm{GC}(\mathrm{HR}=0.57,95 \% \mathrm{CI}=$ $0.35-0.93, P=2.1 \mathrm{E}-02)$. The different effects of SIRT5 on $\mathrm{OS}$ in $\mathrm{GC}$ with different stages or differentiations should be checked in more large cohorts.

Involvement of SIRT7 in GC was reported in only one study that it can promote gastric cancer growth and inhibit apoptosis by epigenetically inhibiting miR-34a and is associated with poor prognosis [51]. Here, we also identified SIRT7 as a biomarker for poor OS in GC $(\mathrm{HR}=$ $1.73,95 \% \mathrm{CI}=1.45-2.05, P=3.2 \mathrm{E}-10)$.

\section{MATERIALS AND METHODS}

To investigate the associations of sirtuins mRNA levels with clinicopathological parameters, we downloaded gastric cancer mRNA profile data and corresponding clinical data from publicly available GEO database. GSE62254 and GSE15459 with large number of gastric cancer patient samples and complete clinical information were selected, which had been also included in the Kaplan-Meier plotter database. The clinical samples were stratified into two groups by sirtuins mRNA levels with the median as cut-off value. The Chi-square test or Fisher's exact test were performed to explore the correlations between sirtuins expression and clinicopathological parameters. Statistical analyses were conducted with the software GraphPad Prism 6. $P$ value less than 0.05 was considered statistically significant.

Then we used the Kaplan-Meier plotter (www. kmplot.com) to investigate the predictive value of mRNA expressions of sirtuins in overall survival in gastric cancer. Currently, the Kaplan-Meier plotter is capable 
Table 7: Correlations of sirtuins with OS in GC stratified by lauren classification

\begin{tabular}{|c|c|c|c|c|c|}
\hline Sirtuins & Lauren classification & Cases & HR & $95 \% \mathrm{CI}$ & $P$-value \\
\hline \multirow[t]{3}{*}{$\begin{array}{l}\text { SIRT1 } \\
\end{array}$} & Intestinal & 320 & 0.51 & $0.37-0.70$ & 2.1E-05 \\
\hline & Diffuse & 141 & 1.26 & $0.88-1.81$ & 2.1E-01 \\
\hline & Mixed & 32 & 0.43 & $0.15-1.22$ & $1.0 \mathrm{E}-01$ \\
\hline \multirow[t]{3}{*}{ SIRT2 } & Intestinal & 320 & 3.39 & $2.32-4.95$ & 2.4E-11 \\
\hline & Diffuse & 141 & 1.71 & $1.17-2.51$ & $5.4 \mathrm{E}-03$ \\
\hline & Mixed & 32 & 2.02 & $0.57-7.23$ & 2.7E-01 \\
\hline \multirow[t]{3}{*}{ SIRT3 } & Intestinal & 320 & 2.74 & $1.89-3.96$ & 2.4E-08 \\
\hline & Diffuse & 141 & 1.44 & $1.01-2.04$ & 4.3E-02 \\
\hline & Mixed & 32 & 4.07 & $1.14-14.47$ & $1.9 \mathrm{E}-02$ \\
\hline \multirow[t]{3}{*}{ SIRT4 } & Intestinal & 320 & 1.37 & $1.00-1.88$ & 4.8E-02 \\
\hline & Diffuse & 141 & 1.59 & $1.13-2.24$ & 7.1E-03 \\
\hline & Mixed & 32 & 2.42 & $0.86-6.85$ & $8.6 \mathrm{E}-02$ \\
\hline \multirow[t]{3}{*}{ SIRT5 } & Intestinal & 169 & 1.32 & $0.90-1.93$ & $1.5 \mathrm{E}-01$ \\
\hline & Diffuse & 140 & 0.79 & $0.56-1.12$ & $1.8 \mathrm{E}-01$ \\
\hline & Mixed & 29 & 0.37 & $0.11-1.20$ & $8.4 \mathrm{E}-02$ \\
\hline \multirow[t]{3}{*}{ SIRT6 } & Intestinal & 320 & 3.03 & $2.05-4.48$ & 4.9E-09 \\
\hline & Diffuse & 141 & 1.38 & $0.98-1.95$ & $6.8 \mathrm{E}-02$ \\
\hline & Mixed & 32 & 2.31 & $0.77-6.94$ & $1.2 \mathrm{E}-01$ \\
\hline \multirow[t]{3}{*}{ SIRT7 } & Intestinal & 320 & 2.71 & $1.91-3.83$ & 5.1E-09 \\
\hline & Diffuse & 141 & 1.37 & $0.97-1.92$ & 7.1E-02 \\
\hline & Mixed & 32 & 2.40 & $0.83-6.95$ & $9.7 \mathrm{E}-02$ \\
\hline
\end{tabular}

Table 8: Correlations of sirtuins with OS in GC stratified by differentiation

\begin{tabular}{llcccc}
\hline Sirtuins & Differentiation & Cases & HR & $\mathbf{9 5 \%}$ CI & P-value \\
\hline SIRT1 & Poor & 165 & 0.71 & $0.46-1.10$ & $1.2 \mathrm{E}-01$ \\
& Moderate & 67 & 0.62 & $0.32-1.21$ & $1.6 \mathrm{E}-01$ \\
SIRT2 & Well & 32 & 0.50 & $0.19-1.30$ & $1.5 \mathrm{E}-01$ \\
& Poor & 165 & 0.72 & $0.48-1.10$ & $1.3 \mathrm{E}-01$ \\
& Moderate & 67 & 1.43 & $0.72-2.83$ & $3.1 \mathrm{E}-01$ \\
SIRT3 & Well & 32 & 0.44 & $0.15-1.31$ & $1.3 \mathrm{E}-01$ \\
& Poor & 165 & 1.24 & $0.83-1.85$ & $2.9 \mathrm{E}-01$ \\
& Moderate & 67 & $\mathbf{0 . 4 7}$ & $\mathbf{0 . 2 5}-\mathbf{0 . 9 0}$ & $\mathbf{2 . 0 E}-\mathbf{0 2}$ \\
SIRT4 & Well & 32 & $\mathbf{3 . 8 9}$ & $\mathbf{1 . 5 9 - 9 . 4 9}$ & $\mathbf{1 . 5 E}-\mathbf{0 3}$ \\
& Poor & 165 & $\mathbf{1 . 7 9}$ & $\mathbf{1 . 1 9}-\mathbf{2 . 6 8}$ & $\mathbf{4 . 2 E}-\mathbf{0 3}$ \\
& Moderate & 67 & 0.56 & $0.27-1.14$ & $1.0 \mathrm{E}-01$ \\
SIRT5 & Well & 32 & 0.45 & $0.18-1.15$ & $8.8 \mathrm{E}-02$ \\
& Poor & 121 & $\mathbf{0 . 5 7}$ & $\mathbf{0 . 3 5}-\mathbf{0 . 9 3}$ & $\mathbf{2 . 1 E}-\mathbf{0 2}$ \\
SIRT6 & Moderate & 67 & 1.42 & $0.72-2.81$ & $3.1 \mathrm{E}-01$ \\
& Well & 5 & NA & & \\
& Poor & 165 & 0.75 & $0.49-1.12$ & $1.6 \mathrm{E}-01$ \\
SIRT7 & Moderate & 67 & 1.75 & $0.9-3.42$ & $9.5 \mathrm{E}-02$ \\
& Well & 32 & 0.62 & $0.25-1.55$ & $3.1 \mathrm{E}-01$ \\
& Poor & 165 & 1.37 & $0.88-2.12$ & $1.6 \mathrm{E}-01$ \\
& Moderate & 67 & 1.61 & $0.67-3.88$ & $2.8 \mathrm{E}-01$ \\
& Well & 32 & $\mathbf{0 . 3 0}$ & $\mathbf{0 . 1 0}-\mathbf{0 . 9 0}$ & $\mathbf{2 . 3 E}-\mathbf{0 2}$ \\
\hline
\end{tabular}


Table 9: Correlations of sirtuins with OS in GC stratified by treatment

\begin{tabular}{llcccc}
\hline Sirtuins & Treatment & Cases & HR & $\mathbf{9 5 \%}$ CI & $\boldsymbol{P}$-value \\
\hline SIRT1 & Surgery alone & 380 & 1.20 & $0.89-1.62$ & $2.4 \mathrm{E}-01$ \\
& 5-FU adjuvant & 153 & $\mathbf{0 . 6 4}$ & $\mathbf{0 . 4 4 - 0 . 9 5}$ & $\mathbf{2 . 4 E}-\mathbf{0 2}$ \\
& Other adjuvant & 76 & 1.99 & $0.72-5.47$ & $1.8 \mathrm{E}-01$ \\
SIRT2 & Surgery alone & 380 & $\mathbf{1 . 5 5}$ & $\mathbf{1 . 1 5}-\mathbf{2 . 0 8}$ & $\mathbf{3 . 7 E}-\mathbf{0 3}$ \\
& 5-FU adjuvant & 153 & $\mathbf{0 . 6 8}$ & $\mathbf{0 . 4 8}-\mathbf{0 . 9 6}$ & $\mathbf{2 . 8 E}-\mathbf{0 2}$ \\
& Other adjuvant & 76 & 6.10 & $1.41-26.33$ & $5.7 \mathrm{E}-03$ \\
SIRT3 & Surgery alone & 380 & $\mathbf{1 . 4 7}$ & $\mathbf{1 . 0 9}-\mathbf{1 . 9 7}$ & $\mathbf{1 . 1 E}-\mathbf{0 2}$ \\
& 5-FU adjuvant & 153 & 0.71 & $0.48-1.05$ & $8.3 \mathrm{E}-02$ \\
& Other adjuvant & 76 & 1.66 & $0.68-4.05$ & $2.6 \mathrm{E}-01$ \\
SIRT4 & Surgery alone & 380 & $\mathbf{1 . 3 7}$ & $\mathbf{1 . 0 2}-\mathbf{1 . 8 2}$ & $\mathbf{3 . 3 E}-\mathbf{0 2}$ \\
& 5-FU adjuvant & 153 & 1.25 & $0.85-1.84$ & $2.5 \mathrm{E}-01$ \\
& Other adjuvant & 76 & 0.60 & $0.22-1.66$ & $3.2 \mathrm{E}-01$ \\
SIRT5 & Surgery alone & 380 & 1.26 & $0.94-1.69$ & $1.2 \mathrm{E}-01$ \\
& 5-FU adjuvant & 34 & 0.23 & $0.08-0.68$ & $3.7 \mathrm{E}-03$ \\
& Other adjuvant & 76 & 0.75 & $0.31-1.80$ & $5.1 \mathrm{E}-01$ \\
SIRT6 & Surgery alone & 380 & 1.33 & $1.00-1.79$ & $5.2 \mathrm{E}-02$ \\
& 5-FU adjuvant & 153 & 1.26 & $0.89-1.79$ & $1.9 \mathrm{E}-01$ \\
& Other adjuvant & 76 & $\mathbf{0 . 3 6}$ & $\mathbf{0 . 1 5}-\mathbf{0 . 8 8}$ & $\mathbf{2 . 0 E}-\mathbf{0 2}$ \\
SIRT7 & Surgery alone & 380 & $\mathbf{1 . 3 5}$ & $\mathbf{1 . 0 1}-\mathbf{1 . 8 0}$ & $\mathbf{4 . 3 E - 0 2}$ \\
& 5-FU adjuvant & 153 & $\mathbf{1 . 6 0}$ & $\mathbf{1 . 1 2}-\mathbf{2 . 2 7}$ & $\mathbf{8 . 4 E - 0 3}$ \\
& Other adjuvant & 76 & 0.59 & $0.24-1.43$ & $2.4 \mathrm{E}-01$ \\
\hline
\end{tabular}

Table 10: Correlations of sirtuins with OS in GC stratified by HER2 status

\begin{tabular}{llllll}
\hline Sirtuins & HER2 status & Cases & HR & $\mathbf{9 5 \%}$ CI & $\boldsymbol{P}$-value \\
\hline SIRT1 & Negative & 532 & $\mathbf{0 . 6 4}$ & $\mathbf{0 . 5 1 - 0 . 8 1}$ & $\mathbf{1 . 4 E - 0 4}$ \\
& Positive & 344 & $\mathbf{0 . 5 8}$ & $\mathbf{0 . 4 2 - 0 . 8 0}$ & $\mathbf{7 . 3 E - 0 4}$ \\
SIRT2 & Negative & 532 & $\mathbf{2 . 2 0}$ & $\mathbf{1 . 7 1 - 2 . 8 3}$ & $\mathbf{3 . 9 E}-\mathbf{1 0}$ \\
& Positive & 344 & $\mathbf{1 . 5 8}$ & $\mathbf{1 . 1 4 - 2 . 1 8}$ & $\mathbf{5 . 5 E}-\mathbf{0 3}$ \\
SIRT3 & Negative & 532 & $\mathbf{1 . 6 8}$ & $\mathbf{1 . 3 1 - 2 . 1 5}$ & $\mathbf{3 . 2 E}-\mathbf{0 5}$ \\
& Positive & 344 & $\mathbf{1 . 5 2}$ & $\mathbf{1 . 1 1}-\mathbf{2 . 0 6}$ & $\mathbf{7 . 7 E - 0 3}$ \\
SIRT4 & Negative & 532 & $\mathbf{1 . 6 0}$ & $\mathbf{1 . 2 7 - 2 . 0 0}$ & $\mathbf{4 . 7 E - 0 5}$ \\
& Positive & 344 & 1.28 & $0.98-1.67$ & $7.0 \mathrm{E}-02$ \\
SIRT5 & Negative & 429 & 0.83 & $0.62-1.12$ & $2.2 \mathrm{E}-01$ \\
& Positive & 202 & 0.63 & $\mathbf{0 . 4 3 - 0 . 9 4}$ & $\mathbf{2 . 2 E}-\mathbf{0 2}$ \\
SIRT6 & Negative & 532 & 1.81 & $\mathbf{1 . 4 3 - 2 . 2 9}$ & $\mathbf{5 . 2 E}-\mathbf{0 7}$ \\
& Positive & 344 & 1.76 & $\mathbf{1 . 2 8}-\mathbf{2 . 4 1}$ & $\mathbf{3 . 8 E}-\mathbf{0 4}$ \\
SIRT7 & Negative & 532 & 1.91 & $\mathbf{1 . 5 3 - 2 . 4 0}$ & $\mathbf{1 . 0 E}-\mathbf{0 8}$ \\
& Positive & 344 & 1.60 & $\mathbf{1 . 2 3 - 2 . 0 7}$ & $\mathbf{3 . 8 E}-\mathbf{0 4}$ \\
\hline
\end{tabular}

to assess the effect of 54, 675 genes on survival of 10 , 188 clinical cancer samples, including 4, 142 breast, 1, 648 ovarian, 2, 437 lung and 1, 065 gastric cancer patients[52]. Briefly, the seven sirtuins (SIRT1, SIRT2, SIRT3, SIRT4, SIRT5, SIRT6, and SIRT7) were entered into the database (http://kmplot.com/analysis/index. php?p 
$=$ service and cancer $=$ gastric) to obtain Kaplan-Meier plots in which the number-at-risk is indicated below the main plot. The Affy IDs of SIRT1-7 were 218878_s_at (SIRT1), 220605_s_at (SIRT2), 221913_at (SIRT3), 220047_at (SIRT4), 229112_at (SIRT5), 219613_s_at (SIRT6), and 218797_s_at (SIRT7), respectively. If the gene had multiple chip probe sets, JetSet best probe set was selected. The patient samples were divided into two groups according to the mRNA expression with auto select best cutoff value (high vs. low expression). The hazard ratio (HR) with $95 \%$ confidence intervals and log rank $p$ value was calculated and displayed on the webpage. HER2 status was determined using the gene chip probe set 216836_s_at as described before [21].

\section{CONCLUSIONS}

To investigate and compare the clinical value of sirtuins in predicting overall survival in GC, we analyze the associations of all sirtuins (SIRT1-7) mRNA expressions with overall survival in GC using an online database, KM plotter. And the results suggested that high SIRT1 mRNA level was associated with better overall survival, SIRT2-4 and 6-7 were associated with poor overall survival, whereas SIRT5 did not show significant association with overall survival in GC.

\section{Author contributions}

Designed the study: XS and PL. Searched databases: PL, YX, and XC. Extracted and analyzed the data: YX, XC, HS, and YZ. Statistical analyses: XS, ML, and WZ. Wrote the main manuscript text: XS and PL. All authors reviewed the manuscript.

\section{ACKNOWLEDGMENTS}

None.

\section{CONFLICTS OF INTEREST}

The authors declare no conflicts of interest.

\section{GRANT SUPPORT}

This study was supported by the grants from the National Natural Science Foundation of China (No.81472940).

\section{REFERENCES}

1. Jemal A, Siegel R, Ward E, Hao Y, Xu J, Murray T, Thun MJ. Cancer statistics, 2008. CA Cancer J Clin. 2008; 58:71-96.

2. Siegel R, Ma J, Zou Z, Jemal A. Cancer statistics, 2014. CA Cancer J Clin. 2014; 64:9-29.
3. Kamangar F, Dores GM, Anderson WF. Patterns of cancer incidence, mortality, and prevalence across five continents: defining priorities to reduce cancer disparities in different geographic regions of the world. J Clin Oncol. 2006; 24:2137-2150.

4. Bang YJ, Van Cutsem E, Feyereislova A, Chung HC, Shen L, Sawaki A, Lordick F, Ohtsu A, Omuro Y, Satoh T, Aprile G, Kulikov E, Hill J, et al. Trastuzumab in combination with chemotherapy versus chemotherapy alone for treatment of HER2-positive advanced gastric or gastrooesophageal junction cancer (ToGA): a phase 3, open-label, randomised controlled trial. Lancet. 2010; 376:687-697.

5. Lee J, Lim DH, Kim S, Park SH, Park JO, Park YS, Lim HY, Choi MG, Sohn TS, Noh JH, Bae JM, Ahn YC, Sohn I, et al. Phase III trial comparing capecitabine plus cisplatin versus capecitabine plus cisplatin with concurrent capecitabine radiotherapy in completely resected gastric cancer with D2 lymph node dissection: the ARTIST trial. J Clin Oncol. 2012; 30:268-273.

6. Ali Z, Deng Y, Tang Y, Zheng S, Ma N, He N. Epigenetic deregulations in gastric cancer. J Nanosci Nanotechnol. 2013; 13:40-51.

7. Imai S, Armstrong CM, Kaeberlein M, Guarente L. Transcriptional silencing and longevity protein Sir2 is an NAD-dependent histone deacetylase. Nature. 2000; 403:795-800.

8. Landry J, Sutton A, Tafrov ST, Heller RC, Stebbins J, Pillus L, Sternglanz R. The silencing protein SIR2 and its homologs are NAD-dependent protein deacetylases. Proc Natl Acad Sci USA. 2000; 97:5807-5811.

9. North BJ, Marshall BL, Borra MT, Denu JM, Verdin E. The human Sir2 ortholog, SIRT2, is an NAD+-dependent tubulin deacetylase. Mol Cell. 2003; 11:437-444.

10. Tanner KG, Landry J, Sternglanz R, Denu JM. Silent information regulator 2 family of NAD- dependent histone/ protein deacetylases generates a unique product, 1-O-acetylADP-ribose. Proc Natl Acad Sci USA. 2000; 97:1417814182.

11. Haigis MC, Mostoslavsky R, Haigis KM, Fahie K, Christodoulou DC, Murphy AJ, Valenzuela DM, Yancopoulos GD, Karow M, Blander G, Wolberger C, Prolla TA, Weindruch R, et al. SIRT4 inhibits glutamate dehydrogenase and opposes the effects of calorie restriction in pancreatic beta cells. Cell. 2006; 126:941-954.

12. Liszt G, Ford E, Kurtev M, Guarente L. Mouse Sir2 homolog SIRT6 is a nuclear ADP-ribosyltransferase. J Biol Chem. 2005; 280:21313-21320.

13. Barber MF, Michishita-Kioi E, Xi Y, Tasselli L, Kioi M, Moqtaderi Z, Tennen RI, Paredes S, Young NL, Chen K, Struhl K, Garcia BA, Gozani O, et al. SIRT7 links H3K18 deacetylation to maintenance of oncogenic transformation. Nature. 2012; 487:114-118.

14. Michishita E, McCord RA, Boxer LD, Barber MF, Hong T, Gozani O, Chua KF. Cell cycle-dependent deacetylation 
of telomeric histone H3 lysine K56 by human SIRT6. Cell Cycle. 2009; 8:2664-2666.

15. Du J, Zhou Y, Su X, Yu JJ, Khan S, Jiang H, Kim J, Woo J, Kim JH, Choi BH, He B, Chen W, Zhang S, et al. Sirt5 is a NAD-dependent protein lysine demalonylase and desuccinylase. Science. 2011; 334:806-809.

16. Houtkooper RH, Pirinen E, Auwerx J. Sirtuins as regulators of metabolism and healthspan. Nat Rev Mol Cell Biol. 2012; 13:225-238.

17. Bonda DJ, Lee HG, Camins A, Pallas M, Casadesus G, Smith MA, Zhu X. The sirtuin pathway in ageing and Alzheimer disease: mechanistic and therapeutic considerations. Lancet Neurol. 2011; 10:275-279.

18. Borradaile NM, Pickering JG. NAD $(+)$, sirtuins, and cardiovascular disease. Curr Pharm Des. 2009; 15:110-117.

19. Hall JA, Dominy JE, Lee Y, Puigserver P. The sirtuin family's role in aging and age-associated pathologies. J Clin Invest. 2013; 123:973-979.

20. Gyorffy B, Lanczky A, Eklund AC, Denkert C, Budczies J, Li Q, Szallasi Z. An online survival analysis tool to rapidly assess the effect of 22,277 genes on breast cancer prognosis using microarray data of 1,809 patients. Breast Cancer Res Treat. 2010; 123:725-731.

21. Gyorffy B, Benke Z, Lanczky A, Balazs B, Szallasi Z, Timar J, Schafer R. RecurrenceOnline: an online analysis tool to determine breast cancer recurrence and hormone receptor status using microarray data. Breast Cancer Res Treat. 2012; 132:1025-1034.

22. Cha EJ, Noh SJ, Kwon KS, Kim CY, Park BH, Park HS, Lee H, Chung MJ, Kang MJ, Lee DG, Moon WS, Jang KY. Expression of DBC1 and SIRT1 is associated with poor prognosis of gastric carcinoma. Clin Cancer Res. 2009; 15:4453-4459.

23. Feng AN, Zhang LH, Fan XS, Huang Q, Ye Q, Wu HY, Yang J. Expression of SIRT1 in gastric cardiac cancer and its clinicopathologic significance. Int J Surg Pathol. 2011; 19:743-750.

24. Zhang LH, Huang Q, Fan XS, Wu HY, Yang J, Feng AN. Clinicopathological significance of SIRT1 and p300/CBP expression in gastroesophageal junction (GEJ) cancer and the correlation with E-cadherin and MLH1. Pathol Res Pract. 2013; 209:611-617.

25. Noguchi A, Kikuchi K, Zheng H, Takahashi H, Miyagi Y, Aoki I, Takano Y. SIRT1 expression is associated with a poor prognosis, whereas $\mathrm{DBC} 1$ is associated with favorable outcomes in gastric cancer. Cancer Med. 2014; 3:1553-1561.

26. Kang Y, Jung WY, Lee H, Lee E, Kim A, Kim BH. Expression of SIRT1 and DBC1 in Gastric Adenocarcinoma. Korean J Pathol. 2012; 46:523-531.

27. Jiang B, Chen JH, Yuan WZ, Ji JT, Liu ZY, Wu L, Tang Q, Shu XG. Prognostic and clinical value of Sirt1 expression in gastric cancer: A systematic meta-analysis. J Huazhong Univ Sci Technolog Med Sci. 2016; 36:278-284.
28. Cao YW, Li YC, Wan GX, Du XM, Li F. Clinicopathological and prognostic role of SIRT1 in breast cancer patients: a meta-analysis. Int J Clin Exp Med. 2015; 8:616-624.

29. Jiang H, Zhang X, Tao Y, Shan L, Jiang Q, Yu Y, Cai F, Ma L. Prognostic and clinicopathologic significance of SIRT1 expression in hepatocellular carcinoma. Oncotarget. 2016; 8:52357-52365. https://doi.org/10.18632/oncotarget.14096.

30. Zu G, Ji A, Zhou T, Che N. Clinicopathological significance of SIRT1 expression in colorectal cancer: A systematic review and meta analysis. Int J Surg. 2016; 26:32-37.

31. Chen Y, Wang T, Wang W, Hu J, Li R, He S, Yang J. Prognostic and clinicopathological significance of SIRT1 expression in NSCLC: a meta-analysis. Oncotarget. 2017; 8:62537-62544. https://doi.org/10.18632/oncotarget.19244.

32. Wang C, Yang W, Dong F, Guo Y, Tan J, Ruan S, Huang T. The prognostic role of Sirt1 expression in solid malignancies: a meta-analysis. Oncotarget. 2017; 8:6634366351. https://doi.org/10.18632/oncotarget.18494.

33. Lu J, Zhang L, Chen X, Lu Q, Yang Y, Liu J, Ma X. SIRT1 counteracted the activation of STAT3 and NF-kappaB to repress the gastric cancer growth. Int J Clin Exp Med. 2014; 7:5050-5058.

34. Yang Q, Wang B, Gao W, Huang S, Liu Z, Li W, Jia J. SIRT1 is downregulated in gastric cancer and leads to G1-phase arrest via NF-kappaB/Cyclin D1 signaling. Mol Cancer Res. 2013; 11:1497-1507.

35. Li J, Dong G, Wang B, Gao W, Yang Q. miR-543 promotes gastric cancer cell proliferation by targeting SIRT1. Biochem Biophys Res Commun. 2016; 469:15-21.

36. Zhang L, Wang X, Chen P. MiR-204 down regulates SIRT1 and reverts SIRT1-induced epithelial-mesenchymal transition, anoikis resistance and invasion in gastric cancer cells. BMC Cancer. 2013; 13:290.

37. Peters CJ, Rees JR, Hardwick RH, Hardwick JS, Vowler SL, Ong CA, Zhang C, Save V, O'Donovan M, Rassl D, Alderson D, Caldas C, Fitzgerald RC. A 4-gene signature predicts survival of patients with resected adenocarcinoma of the esophagus, junction, and gastric cardia. Gastroenterology. 2010; 139:1995-2004 e1915.

38. Huang KH, Hsu CC, Fang WL, Chi CW, Sung MT, Kao HL, Li AF, Yin PH, Yang MH, Lee HC. SIRT3 expression as a biomarker for better prognosis in gastric cancer. World J Surg. 2014; 38:910-917.

39. Yang B, Fu X, Shao L, Ding Y, Zeng D. Aberrant expression of SIRT3 is conversely correlated with the progression and prognosis of human gastric cancer. Biochem Biophys Res Commun. 2014; 443:156-160.

40. Hur H, Kim YB, Ham IH, Lee D. Loss of ACSS2 expression predicts poor prognosis in patients with gastric cancer. $\mathrm{J}$ Surg Oncol. 2015; 112:585-591.

41. Yu FY, Xu Q, Wu DD, Lau AT, Xu YM. The Prognostic and Clinicopathological Roles of Sirtuin-3 in Various Cancers. PLoS One. 2016; 11:e0159801. 
42. Wang L, Wang WY, Cao LP. SIRT3 inhibits cell proliferation in human gastric cancer through down-regulation of Notch-1. Int J Clin Exp Med. 2015; 8:5263-5271.

43. Cui Y, Qin L, Wu J, Qu X, Hou C, Sun W, Li S, Vaughan AT, Li JJ, Liu J. SIRT3 Enhances Glycolysis and Proliferation in SIRT3-Expressing Gastric Cancer Cells. PLoS One. 2015; 10:e0129834.

44. Huang G, Cui F, Yu F, Lu H, Zhang M, Tang H, Peng Z. Sirtuin-4 (SIRT4) is downregulated and associated with some clinicopathological features in gastric adenocarcinoma. Biomed Pharmacother. 2015; 72:135-139.

45. Bai LH, Lin G, Sun L, Liu Y, Huang X, Cao C, Guo Y, Xie C. Upregulation of SIRT6 predicts poor prognosis and promotes metastasis of non-small cell lung cancer via the ERK1/2/MMP9 pathway. Oncotarget. 2016; 7:4037740386. https://doi.org/10.18632/oncotarget.9750.

46. Lu CT, Hsu CM, Lin PM, Lai CC, Lin HC, Yang CH, Hsiao HH, Liu YC, Lin HY, Lin SF, Yang MY. The potential of SIRT6 and SIRT7 as circulating markers for head and neck squamous cell carcinoma. Anticancer Res. 2014; 34:71377143 .

47. Marquardt JU, Fischer K, Baus K, Kashyap A, Ma S, Krupp M, Linke M, Teufel A, Zechner U, Strand D, Thorgeirsson
SS, Galle PR, Strand S. Sirtuin-6-dependent genetic and epigenetic alterations are associated with poor clinical outcome in hepatocellular carcinoma patients. Hepatology. 2013; 58:1054-1064.

48. Zhang G, Liu Z, Qin S, Li K. Decreased expression of SIRT6 promotes tumor cell growth correlates closely with poor prognosis of ovarian cancer. Eur J Gynaecol Oncol. 2015; 36:629-632.

49. Igci M, Kalender ME, Borazan E, Bozgeyik I, Bayraktar R, Bozgeyik E, Camci C, Arslan A. High-throughput screening of Sirtuin family of genes in breast cancer. Gene. 2016; 586:123-128.

50. Lu W, Zuo Y, Feng Y, Zhang M. SIRT5 facilitates cancer cell growth and drug resistance in non-small cell lung cancer. Tumour Biol. 2014; 35:10699-10705.

51. Zhang S, Chen P, Huang Z, Hu X, Chen M, Hu S, Hu Y, Cai T. Sirt7 promotes gastric cancer growth and inhibits apoptosis by epigenetically inhibiting miR-34a. Sci Rep. 2015; 5:9787.

52. Gyorffy B, Surowiak P, Budczies J, Lanczky A. Online survival analysis software to assess the prognostic value of biomarkers using transcriptomic data in non-small-cell lung cancer. PLoS One. 2013; 8:e82241. 